\title{
X. In Catholicis
}

Zunächst muß hier noch einmal auf die frühen sechziger Jahre zurückgegriffen werden. Schauffs gute Dienste bei dem Versuch, den deutschen Sozialdemokraten ein Entrée im Vatikan zu verschaffen, waren zeitlich und inhaltlich mit seinem Engagement bei dem 1961 beginnenden Zweiten Vatikanischen Konzil zusammengefallen. Durch seine Tätigkeit für die ICMC, aber auch für die christdemokratische Fédération Internationale, waren ihm Rom wohlvertraut und die wichtigsten Repräsentanten der Kurie persönlich bekannt. Der Wunsch, bei diesem Treffen der Weltkirche präsent zu sein und nach Möglichkeit aktiv zu werden, motivierten ihn dazu, seinen Wohnsitz in Rom zu nehmen. Rom war ja die erste Station der Familie nach der Emigration gewesen. Die Schauffs bezogen nunmehr eine Wohnung in der Via Gregoriana, am Abhang des Pincio und in unmittelbarer Nähe der Spanischen Treppe.

Lage und Größe des neuen Refugiums waren von vornherein auch unter dem Gesichtspunkt getroffen worden, daß es als Begegnungsstätte von Mitgliedern und interessierten Beobachtern des Konzils dienen konnte. Im Verlauf des Konzils waren dies 1500 bis 2000 Besucher: Konzilsväter aus Südamerika, Deutschland und Polen, junge Theologen und Publizisten aus aller Welt, Mitarbeiter internationaler Organisationen sowie nichtkatholische und ökumenisch orientierte Konzilsbeobachter. Karin Schauff hatte als Hausherrin in diesem Zusammenhang immense Arbeit zu leisten ${ }^{1}$. All diese Aktivitäten sowie Schauffs Bekanntheitsgrad als Repräsentant internationaler katholischer Organisationen trugen zu seiner späteren Berufung in die postkonziliare Kurienkommission "Justitia et Pax“ wesentlich bei.

\section{Das Zweite Vatikanische Konzil: Schauffs Berufung in die Kurienkommission "Justitia et Pax“}

Das Zweite Vatikanische Konzil wurde von Papst Johannes XXIII. am 25. Dezember 1961 einberufen, nach seinem Tode ab Juni 1963 von dem Nachfolger Paul VI. weitergeführt und am 8 . Dezember 1965 beendet $^{2}$. Zentrales Thema war der notwendige Wandel der Kirche, ihrer Organisation und ihrer Seelsorgemethoden in einer zutiefst sich wandelnden Welt, das von Papst Johannes so genannte "aggiornamento".

Anders als das Konzil während des Pontifikats von Pius IX. im Jahre 1869/70 war das Zweite Vatikanum ein Weltereignis und fand eine entsprechende öffentliche Resonanz. In vier Sitzungsperioden wurden 168 Generalkongregationen

1 Vgl. Karin Schauff, Wahlheimat Rom, S. 24 ff.; Um der Freiheit willen, S. 33; Herwarth, Adenauer, S. $315 \mathrm{f}$.

2 Das Zweite Vatikanische Konzil; weiterführende Literatur bei Jedin, Konzil, S. $97 \mathrm{ff}$. 
(Plenarsitzungen) und dazu zehn öffentliche Sitzungen abgehalten. Die erarbeiteten Ergebnisse und Aussagen sind in 16 Texten niedergelegt, worunter das Dekret über das Laienapostolat „Apostolicam actuositatem“ und die umfangreiche $\mathrm{Pa}$ storalkonstitution über die Kirche in der heutigen Welt, "Gaudium et Spes“, das eigentliche Herzstück der Konzilsarbeit, wesentliche Grundlagen für die postkonziliare Arbeit bildeten, in die Johannes Schauff eingebunden wurde.

Das Zentralthema - die Kirche in einer sich wandelnden Welt - lenkte den Blick alsbald auf die gravierenden materiellen und kulturellen Entwicklungsprobleme der sogenannten Dritten Welt. Insbesondere Dom Helder Câmara, zu dieser Zeit Weihbischof von Rio de Janeiro, machte das Konzil auf die Probleme der Unterentwicklung und des Hungers aufmerksam. Zusammen mit einer Gruppe um den französischen Abbé Paul Gauthier wurde Kardinal Cicognani die Notwendigkeit eines eigenen Sekretariats in dieser Frage vermittelt. Von Kardinal Suenens wurde die Unterscheidung getroffen, die Beratungen über den Standort der Kirche nicht nur "ad intra“, sondern angesichts der Probleme der Dritten Welt auch „ad extra“ zu strukturieren. Dabei sollte die Wahrnehmung der Kirche als einer Kirche der Armen geschärft werden ${ }^{3}$.

Am 5. Juli 1966 wurde Schauff durch Staatssekretär Kardinal Cicognani in das Vorbereitungskomitee zur Gründung nachkonziliarer Instanzen berufen, das auf der Grundlage des Konzildekrets „Apostolicam actuositatem“ (Artikel 26) und der Pastoralkonstitution „Gaudium et Spes“ (Artikel 90) tätig werden sollte. Vorsitzender dieses Vorbereitungskomitees war der kanadische Kardinal und Bischof von Quebec, Maurice Roy ${ }^{4}$.

Bereits in den Artikeln 86 („De quibus opportunis normis“) und 89 („De praesentia efficaci Ecclesiae in communitati internationali“) von "Gaudium et Spes“ werden die Verpflichtungen der hochentwickelten Länder und der internationalen Gemeinschaft gegenüber den Entwicklungsländern sowie die Notwendigkeit der Zusammenarbeit betont. In Artikel 90 („De partibus christianorum in institutionibus internationalibus“) wird zur Förderung dieser Zusammenarbeit unter den Nationen auf die Notwendigkeit des ,internationalen Wirkens der Christen“ und die Aktionen vor allem der katholischen internationalen Organisationen hingewiesen. Zugleich wird aber auch der ökumenische Aspekt hervorgehoben, die "tatkräftige und positive Zusammenarbeit ... mit den getrennten Brüdern“. Angesichts der zahllosen Drangsale, unter denen ein großer Teil der Menschheit leide, „hält es das Konzil für zweckmäßig, ein Organ der Gesamtkirche zu schaffen, um die Gerechtigkeit und Liebe Christi den Armen in aller Welt zuteil werden zu lassen. Seine Aufgabe soll es sein, die Gemeinschaft der Katholiken immer wieder anzuregen, den Aufstieg der notleidenden Gebiete und die soziale Gerechtigkeit unter den Völkern zu fördern." 5

3 Diese Forderung von seiten des Kardinals Lercaro. Vgl. Charles Moeller, Die Geschichte der Pastoralkonstitution (über die Kirche in der Welt von heute), in: Zweite Vatikanische Konzil, S. 247.

4 IfZ, NL Schauff, Bd. 16.

5 Das Zweite Vatikanische Konzil, Bd. III, S. $569 \mathrm{ff}$. und $576 \mathrm{ff}$. 
In einer solchen religiösen und sozialen Sichtweise mußten naturgemäß gerade die Laien eine wichtige Rolle einnehmen. Dies bringt das Dekret über das Apostolat der Laien in Artikel 26 zum Ausdruck, wo es heißt, daß die Diözesen nach Möglichkeit beratende Gremien einrichten sollten, die die apostolische Tätigkeit der Kirche vor allem im karitativen und sozialen Bereich unterstützen - auf der Ebene der Pfarreien und Diözesen, aber auch im internationalen Bereich ${ }^{6}$.

Allerdings differenzierte die vom Heiligen Stuhl eingesetzte Arbeitsgruppe aus Priestern und Laien zwischen der Laienarbeit und der Aufgabenstellung, die sich aus "Gaudium et Spes" ergab. Bereits im Januar 1966 wurde mit der Einrichtung postkonziliarer Kommissionen auch das Laienapostolat begründet. Wie aus einem Memorandum von Johannes Schauff hervorgeht, sollte „das vom Konzil im Art[ikel] 90 angeregte ,Organ der Gesamtkirche' als eine selbständige Institution errichtet werden ..., ohne Abhängigkeit von der Institution nach

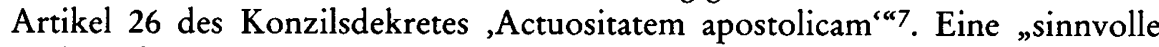
Verknüpfung" der beiden Tätigkeitsbereiche mußte jedoch Gegenstand der Verhandlungen sein.

Schauff sah durchaus das Problem, die in Artikel 90 und den vorausgehenden Artikeln 88 und 89 formulierten Aufgabenstellungen sinnvoll zu verknüpfen: einmal die Koordinierung der praktisch-operativen Tätigkeit von Organisationen, zum andern die Funktion als Organ der Gesamtkirche, das Solidarität und Verantwortung gegenüber notleidenden Gebieten fördern sollte. Um die neue Aufgabenstellung zu strukturieren, hielt er es für wünschenswert, „wenn dazu neben Praktikern und Soziologen mehr Theologen und Professoren der kirchlichen Soziallehre ${ }^{8}$ sowie Kenner der Strukturformen der Weltkirche ... hinzugezogen würden“, daneben Laien mit gründlicher Erfahrung im internationalen Bereich und dessen Organisationen.

Angesichts der Neuartigkeit der Aufgabenstellung plädierte er für einen langsamen organisatorischen Aufbau des neuen Gremiums von unten her, d. h. die Einrichtung von nationalen und regionalen Arbeitsgruppen. Analog zu den päpstlichen Kommissionen sollte einer flexiblen Struktur der Vorzug gegeben werden und Mitglieder nur auf Zeit berufen werden. Bei der Suche nach dem Namen für die neue Kommission warnte Schauff davor, nur ein "Organ für den Frieden" schaffen zu wollen, nachdem der Begriff „Frieden“ von den kommunistischen Staaten einseitig usurpiert worden sei. Gleichwohl sollte das neue Gremium einen Beitrag zur Friedensarbeit leisten und eine eigene Abteilung unter der Leitung von herausragenden Völkerrechtlern, zum Beispiel aus Mitgliedern des Haager Schiedsgerichtshofes, gebildet werden 9 .

6 Ebenda, Bd. II, S. 683.

7 Korrekt: „Apostolicam actuositatem“. Bemerkungen zu dem „Report der Working Group vom 9.-12. 5. 1966“, von Johannes Schauff als Mitglied des "Coetus exsequendos n. 26 Decreti ,A postolicam actuositatem“ et n. 90 Constit. Pastoralis ,Gaudium et Spes““, 1. 9.1966 (IfZ, NL Schauff, Bd. 16).

8 Hier schlug Schauff vor allem Vertreter der deutschen katholischen Soziallehre vor, allen voran Oswald von Nell-Breuning. Vgl. die Korrespondenz Schauff-Kardinal Roy 19731982 (IfZ, NL Schauff, Bd. 22).

9 Schauff, Commissio „Solidaritas Universalis“ ( $\$ 90$ „Gaudium et Spes“). Bemerkungen 
Struktur und personeller Aufbau dieses neuen Gremiums, für das es weder Vorbild noch Erfahrung gab, erforderten nach Schauff einen effizienten Generalsekretär und hauptamtlichen Mitarbeiterstab, zumal der Präsident der Kommission, Kardinal Roy, zugleich dem Laienapostolat vorstand. Drei oder vier Abteilungen seien zu bilden: Neben der Organisationsabteilung für innere Verwaltung sollten Kongresse und Forschungsprojekte mit den Themenschwerpunkten Frieden, Gerechtigkeit und Entwicklung organisiert werden ${ }^{10}$.

Diese Überlegungen Schauffs trugen wesentlich zur definitiven inhaltlichen und organisatorischen Strukturierung der Kommission bei. Der Vorbereitungskommission unter Kardinal Roy, die ihre Arbeit bereits Anfang 1966, d. h. unmittelbar nach Beendigung des Konzils, aufgenommen hatte, gehörten nach Schauffs Berufung in einer dritten, abschließenden Arbeitsphase neben dem unterdessen ernannten Vizepräsidenten Castelli und dem Generalsekretär Achille Glorieux ebenso August Vanistendael, Vittorino Veronese und Rosemary Goldie an. ${ }^{11}$ Vor allem mit Vanistendael, einem Partner Schauffs auch innerhalb der Flüchtlingsarbeit und der FIS, ergab sich eine in vielem ergänzende fruchtbare Zusammenarbeit.

Schließlich erfolgte nach mehreren Monaten intensiver Vorbereitungsarbeit mit dem päpstlichen Motu proprio ${ }^{12}$ vom 6. Januar 1967 - nunmehr unter ihrem definitivem Namen - die Konstituierung der Kurienkommission "Justitia et Pax", gleichzeitig mit der des "Consilium de Laicis“13. Damit wurde die enge Verbindung der beiden Gremien augenfällig, deren gemeinsamer Präsident Kardinal Roy war. Die Laienarbeit war Bestandteil auch der Aktivitäten der Kommission „Justitia et Pax“, die Richtlinien für die Tätigkeit der Laien in der Gegenwart erarbeitete. Dies galt vor allem für die Entwicklungspolitik, die nach Veröffentlichung

zur Namensgebung, Struktur und personellen Zusammensetzung (8.11. 1966), IfZ, NL Schauff, Bd. 16.

10 Schauff entwarf folgendes Schema (NL Schauff, Bd. 16):

I. 1 Präsident

II. 3 Vizepräsidenten

III. 2 Generalsekretäre: 1 Geistlicher und 1 Laie

3 Abteilungsleiter: 1 Geistlicher, 2 Laien

IV. Mitglieder des Vorstands/Kommissionsmitglieder:

6 Mitgl. ex officio

5 Geistliche ad personam

5 Laien ad personam.

Hinzu sollten etwa die gleiche Zahl von „Consultores" kommen.

11 Vittorino Veronese, einer der führenden katholischen Intellektuellen Italiens, war nach 1946 Vertreter seines Landes bei der UNESCO und 1958-61 deren Generaldirektor; ab 1961 Präsident der Banco di Roma, Cameriere Segregato di Spada e Cappa; Konsultor des Sekretariats für die Ungläubigen unter Kardinal König, führend in der Laienbewegung (Präsident der ersten drei Weltkongresse). Erzbischof (tit.) Alberto Castelli (als Vizepräsident) sowie Msgr. Glorieux und Frau Goldie als Sekretär und stellvertretende Sekretärin wurden in den Laienrat berufen.

12 Motu proprio: Gesetzgebungsakte des Papstes, die seiner eigenen Initiative entstammen.

13 Sanctissime domini nostri Pauli divina providentia Papae VI, litterae apostolicae motu proprio datae, quibus Consilium de laicis, et pontificia commissio studiosorum, a "Justitia et Pace“ appellata, constitutur. Rom, 6. 1. 1967 (IfZ, NL Schauff, Bd. 16). 
der Enzyklika „Populorum Progressio“ zu Ostern 1967 einen zentralen Platz in der Aufgabenstellung der Kommission einnahm und zu ihrer großen Charta wurde ${ }^{14}$.

Thema von „Populorum Progressio“ war die Einbindung aller Völker in eine regional, ethnisch und national aufgefächerte, nichtsdestoweniger aber im Sinne eines christlichen Humanismus anzustrebende Gesamtkultur ${ }^{15}$. Dabei richtete die Enzyklika ihr zentrales Augenmerk auf die wirtschaftliche Misere in der Dritten Welt und die Maßnahmen zu deren Überwindung, die, zunächst auf den wirtschaftlichen Bereich konzentriert, zu einer umfassenden kulturellen Entwicklungspolitik ausgeweitet werden sollten.

Gemäß der Aufgabenstellung von "Justitia et Pax“ kam der Kommission - im Sinne einer Katechese - eine Mittlerstellung zwischen dieser Enzyklika und der Welt $\mathrm{zu}^{16}$. Ihr Instrumentarium war die Ausarbeitung handlungsorientierter Studien, deren Zielgruppen einmal die Institutionen und Organisationen der Kirche selbst waren, zum andern die ökumenischen Partner sowie die nichtkonfessionellen internationalen Institutionen. Im ersten Jahr kristallisierten sich folgende Tätigkeitsschwerpunkte heraus, für die entsprechende Arbeitsgruppen bzw. Ausschüsse gebildet wurden.

Ein Tätigkeitsschwerpunkt lautete: Theologie und soziale Orientierung. Weitere waren: wirtschaftliche Entwicklungspolitik unter säkularen Aspekten, beispielsweise die Kampagne zugunsten einer Steuer von einem Prozent des Volkseinkommens für die Entwicklungsländer; gerechtere Lösungen im internationalen Handel und verschiedene ökonomische Projekte im Rahmen der UNO. Dazu kamen unmittelbar kirchliche Operationen wie die Bekämpfung des Analphabetismus oder die Stärkung missionarischer und karitativer Strukturen. Weiter ging es um die internationalen politischen Organisationen "ad construendam pacem" vor allem in Hinblick auf die Wahrung der Menschenrechte, den Kampf gegen den Rassismus sowie um eine weltweite Abrüstung ${ }^{17}$.

Der Rolle von "Justitia et Pax“ im Dialog zwischen der Römischen Kirche und der Welt entsprach, daß Priester und Laien die 14 Kommissionsmitglieder und ebenso viele Konsultoren ${ }^{18}$ stellten. Darunter befanden sich neben den Theologen Fachleute für Wirtschafts- und Agrarfragen, für internationale Politik und Probleme der Migration. Zu diesen gehörte auch Johannes Schauff, dessen Arbeit in

14 Vgl. Stefan Swiezaeski (Professor an der katholischen Universität Lublin und selbst Mitglied der Kurienkommission "Justitia et $\mathrm{Pax}$ “) in seinem Exkurs zur Pastoralkonstitution Artikel 90: Die Kommission "Justitia et Pax“, in: Das Zweite Vatikanische Konzil, Bd. III, S. 579.

15 Vgl. Oswald von Nell-Breuning, Exkurs über „Popolorum Progressio“, in: Das Zweite Vatikanische Konzil, Bd. III, S. $578 \mathrm{f}$.

16 Vgl. Pontifical Commission "Justice and Peace ${ }^{\text {" }}$ - Statement of the Pontifical Commission "Justice and Peace" at the conclusion of its first Planetary Meeting, Vatican City, 25. 4. 1967 (IfZ, NL Schauff, Bd. 18).

17 Audience de Son Eminence le Cardinal Président (Roy) de la Commission Pontificale "Iustitia et Pax“, 12 février 1968 (IfZ, NL Schauff, Bd. 17).

18 Konsultoren: beratende Organe der Kardinalskongregationen. 
der Kommission durch die Berufung von Kollegen aus der ICMC, Msgr. Ligutti und James Norris, fachkundige Unterstützung fand.

Neben dem Präsidenten und Vizepräsidenten - Kardinal Roy und dem spanischen Titularbischof Ramón Torella y Cascante als Nachfolger von Alberto Castelli - wurde ein Generalsekretariat eingerichtet, dessen Leitung Msgr. Joseph Gremillion übernahm. Ihm stand ein sogenanntes Direktionskomitee zur Seite, dem wiederum Schauff, Ligutti, Norris und August Vanistendael angehörten. Übergeordnetes Kommunikationsorgan waren Vollversammlungen von Kommissionsmitgliedern und Konsultoren.

Solche Treffen brachten Ergebnisse in Form von Gesprächsaufzeichnungen und Arbeitsdokumenten, man plante Studienprojekte und deren Förderung und strukturierte die Arbeit von ständigen Ausschüssen ${ }^{19}$. Daneben wurden Anfang 1968 die sogenannten „Tables rondes“ eingerichtet; diese sollten der Koordination der verschiedenen katholischen Organisationen dienen, die international im sozialen Bereich tätig waren ${ }^{20}$ und später (1971) von dem Päpstlichen Rat „Cor unum" abgelöst wurden" ${ }^{21}$.

Einen organisatorisch und inhaltlich wichtigen Schritt machte die Kommission "Justitia et Pax", als in Zusammenarbeit mit den Episkopaten vor Ort nationale Dépendancen in verschiedenen Teilen der Welt und vor allem den Entwicklungsländern eingerichtet wurden 22 . Hier konnte Schauff auf seinen verschiedenen Reisen vor allem nach Lateinamerika als Vertreter der ICMC wie auch der FIS als Informant und Berater von Nutzen sein. So warnte er vor den „Irrlehren der sogenannten politischen oder Befreiungs- oder Revolutionstheologie“, die vor allem in der jungen Generation Anklang fänden ${ }^{23}$. Schauff verfolgte damit weiter die

19 In Schauffs Zeit als Mitglied der Kommission bis 1972 wurden sechs Generalversammlungen abgehalten, die alle in Rom stattfanden, die ersten beiden im April und Oktober 1967 (IfZ, NL Schauff, Bd. 18-21).

20 Schreiben Msgr. Benelli an Generalsekretär Gremillion vom 30. Januar 1968 über die Notwendigkeit der Gründung solcher Tables rondes (IfZ, NL Schauff, Bd. 18). Teilnehmer dieses Runden Tisches waren schließlich u.a. Adveniat, Caritas Internationalis, CIDSE, Laienrat, ICMC, „Justitia et Pax“.

21 Das Gründungsdokument und entsprechende Anschreiben von Benelli an den Vizepräsidenten von "Justitia et Pax", Torella, vom 28. 7. 1971 (IfZ, NL Schauff, Bd. 18).

22 Vgl. die auf der 2. Plenarsitzung (23.-28. 10. 1967) gefaßten Beschlüsse über die Rolle der Kirche in den Entwicklungsländern: Les Commissions nationales (IfZ, NL Schauff, Bd. 18). Zwei europäische Konferenzen der Nationalkommissionen fanden im Mai 1971 in Aachen und im Oktober 1972 in Oostende statt (Protokolle und Analysen in IfZ, NL Schauff, Bd. 21).

$23 \mathrm{Vgl}$. Schreiben Schauff an Kardinal Roy vom 8. 10.1972 (IfZ, NL Schauff, Bd. 22): „Meine Reiseerfahrungen durch Latein- und Nordamerika und Europa in den letzten neun Monaten [meiner] Abwesenheit von Rom sowie die Möglichkeit, in größerer Muße die Literatur zur Thematik studieren zu können ... haben [mich] überzeugt: Die Irrlehren der sogenannten politischen oder Befreiungstheologie haben $\mathrm{m}$. E. einen erschreckenden Umfang vor allem in der jungen Generation angenommen. Diese Verwirrung ist mir noch deutlicher geworden in einem langen Gespräch mit meinem hochbetagten und väterlichen Freund und Mentor, Prof. von Nell-Breuning SJ, in Frankfurt." (IfZ, NL Schauff, Bd. 14); vgl. auch Schreiben Schauff an Pater Oskar Simmel SJ vom 15. 2. 1985, in dem er die Unterstützung der Befreiungstheologie auch durch "Justitia et Pax“ beklagt (IfZ, NL Schauff, Bd. 7), sowie an Bischof Franz Hengsbach vom 16. 2. 1980 (IfZ, NL Schauff, Bd. 34). 
politische Linie einer Zurückdrängung des kommunistischen Einflusses in Lateinamerika, den er bereits in der FIS bekämpft hatte und der seit der Revolution in Kuba in deutlicher Zunahme begriffen war.

Der Erfolg Castros in Kuba hatte viel zu Entwicklung und Bedeutungszunahme einer „Theologie der Revolution“ bzw. der „Befreiungstheologie“ beigetragen, die 1968 auf der lateinamerikanischen Bischofskonferenz in Medellin in Kolumbien auch von Teilen der Kirche sanktioniert wurde. Hinter Schauffs Klage über diese Entwicklung stand jedoch deutlich auch die Verbitterung darüber, daß die lateinamerikanische Befreiungstheologie sich nicht zu Unrecht zumindest formal auf das Zweite Vatikanische Konzil und die Enzyklika „Populorum Progressio" stützen konnte, eine innerkirchliche Entwicklung, zu der Schauff selbst beigetragen hatte: Der hier vorgenommene Neuansatz, die Menschen- und Freiheitsrechte in die päpstliche Soziallehre aufzunehmen, konnte sehr wohl zur Legitimierung revolutionärer Bewegungen dienen ${ }^{24}$.

Auch in der Kurienkommission "Justitia et Pax" bewährte sich Schauff ganz offensichtlich als exzellenter Finanzfachmann ${ }^{25}$. Ein ihm aus seiner Tätigkeit für die ICMC wohlbekanntes Problem stellte sich auch der Kommission und war von Schauff bereits in der Vorbereitungsphase artikuliert worden: Es betraf die Bewegungsfreiheit gegenüber dem vatikanischen Staatssekretariat. Dessen starker Mann hinter dem Staatssekretär, Substitut Msgr. Benelli, versuchte auch die Arbeit von "Justitia et Pax" zu kontrollieren, obwohl die Kommission - anders als die Sekretariate - nach Verfassung und Selbstverständnis ein Organ der Gesamtkirche und nicht ausschließlich der Kurie war ${ }^{26}$. Dem entsprach, daß sämtliche Projekte und Unternehmungen von Initiativen der Kommission selbst und ihrer Mitglieder und Konsultoren ausgingen. In den Konflikten um ausgearbeitete Papiere und Memoranden bewährte sich Schauff häufig als diplomatischer Vermittler ${ }^{27}$.

Die ersten Mitglieder und Konsultatoren von „Justitia et Pax“ waren für fünf Jahre berufen worden. Nach Ablauf dieser „experimentellen“ Aufbauphase, die der Organisation und inhaltlichen Vergewisserung diente, war der Zeitpunkt für ein personelles Revirement gekommen, und auch Schauff verließ auf eigenen Wunsch das Gremium. Zum Jahresende 1971 machte er seinen Abschiedsbesuch bei Msgr. Benelli im Staatssekretariat ${ }^{28}$ und wurde am 23. Juni 1972 offiziell durch

24 Zur Revolutions- bzw. Befreiungstheologie vgl. u.a. Camara, Revolution; Rahner (Hrsg.), Befreiende Theologie; Boff, Liberator.

25 Interview des Verfassers mit August Vanistendael am 15./16. 1. 1993; vgl. u.a. Schauffs "Comments on the financial report" (der Kommission) vom 28. 9. 1971 (IfZ, NL Schauff, Bd. 20 u. 21).

$26 \mathrm{Vgl}$. August Vanistendael, Remarks on the future of the Pontifical commission "Justitia et Pax“ (confidential), Februar 1971 (IfZ, NL Schauff, Bd. 22); ein Beispiel das Schreiben Benellis an den Präsidenten von "Justitia et Pax“, Kardinal Roy, vom 15. 2. 1971 (IfZ, NL Schauff, Bd. 22); Interview des Verfassers mit August Vanistendael, 15./16. 1. 1993.

27 Ebenda.

28 Schauff begründete sein Ausscheiden mit seinem vorgerückten Alter - er stand kurz vor seinem 70. Geburtstag. Vgl. das Schreiben Schauff an den Vizepräsidenten und Geschäftsführer von "Justitia et Pax" vom 15. 9. 1972, IfZ, NL Schauff, Bd. 22. 
Kardinal Villot, seit 1969 Nachfolger von Kardinalstaatssekretär Cicognani, verabschiedet ${ }^{29}$.

29 Schreiben in IfZ, NL Schauff, Bd. 22; ebenso das Abschiedsschreiben der Kommissionskollegen mit pers. Unterschriften vom 20. 9. 1972, verfaßt am ersten Tag der VII. Generalversammlung. 